\title{
Chapter 7: Biases in object location estimation: the role of camera rotations and translation
}

\subsection{General Introduction}

Our ability to navigate and orient critically depends on our ability to recognize the place we are in. Place recognition requires memory for object locations as well as the ability to retrieve object locations from different perspectives (Epstein, Harris, Stanley \& Kanwisher, 1999; Postma, Kessels \& van Asselen, 2004). Typically, place recognition across different perspectives is studied using tasks in which participants are presented with static images depicting a scene, an array of objects or environmental features from one perspective and are then asked to indicate whether the array has changed when presented from a different perspective (Diwadkar \& McNamara, 1997; Schmidt et al., 2007; Hartley et al., 2007; Sulpizio, Committeri, Lambrey, Berthoz, \& Galati, 2013; Montefinese, Sulpizio, Galati \& Committeri, 2015; Muffato, Hilton, Meneghetti, De Beni \& Wiener, 2019; Hilton et al., 2020; Segen, Avraamides, Slattery, Wiener, 2021a, 2021b). Our recent research suggests that such paradigms may yield a systematic bias in reporting memorized locations (Segen, Colombo, Avraamides, Slattery, Wiener, 2021c, 2021d). Specifically, we found that when participants were asked to indicate where an object was positioned after a perspective shift (Segen et al., 2021d) or when asked to judge the direction in which an object has moved after a perspective shift (Segen et al., 2021c), they made systematic errors associated with the direction of the perspective shift. That is, participants appeared to "drag" the object with them during the perspective shift and as a result, shifted their responses further to the direction of the shift. Interestingly, this bias is not driven by distortions introduced in memory as participants also exhibited this bias in perception conditions that did not involve memory (Segen et al., 2021d).

It is not entirely clear what gives rise to this systematic bias. However, given that the

direction of the bias is associated with the direction of the perspective shift, it is likely that the 
bias results from egocentric, rather than allocentric, influences on the estimates. Specifically, we believe that uncertainty about the exact nature of the perspective shift leads to uncertainty about the exact position of the object, which in turn results in participants biasing their estimates towards the egocentric self-to-object estimates derived during encoding (i.e. before the perspective shift). Henceforth, we will refer to this systematic shift in responses in the direction of the perspective shift as the perspective shift related bias. This idea aligns well with the anchoring and adjustment heuristic proposed by Tversky and Kahneman (1974). According to this heuristic, people base their responses on initial estimates (the anchor) that they adjust to correct for errors when they are uncertain. Often, these anchors are based on egocentric representations (Epley, Keysar, Van Boven, Gilovich, 2004; Gilovich, Medvec, \& Savitsky, 2000; Keysar, Barr, Balin, \& Brauner, 2000). Epley et al. (2004), for example, found that participants used their previous experience (exposed either to positive or negative events) when making judgements about how others would perceive ambiguous stimuli (sarcastic or genuine).

In our task (Segen et al., 2021d), participants may use the egocentric vector between themselves and the object during encoding as an anchor for their response. Indeed, using the egocentric representation of the object location as an anchor would result in participants "dragging" the object with them following a perspective shift. In line with this possibility, previous research suggests that adjustments require time and cognitive effort (Epley et al., 2004) and are often insufficient and responses are therefore biased in the direction of the initial anchor, in part, because people stop adjusting once a plausible estimate is reached (Tversky \& Kahneman, 1974; Quattrone, 1982). If participants in our previous experiments (Segen et al., 2021c, 2021d) also showed insufficient adjustments, this would explain why their estimates were systematically biased in the direction of the perspective shift.

Potential sources of uncertainty that may encourage the use of an anchor based on the egocentric self-to-object relations are: (1) uncertainty about the position of the object in 
the environment and (2) difficulties in understanding the exact nature of the perspective shift. The uncertainty about the object position could be reduced by enhancing the environment to include more spatial information i.e. by adding stable environmental cues that help to encode the position of the object more accurately (Cánovas, Leon, Serrano, Roldan \& Cimadevilla, 2011; Chamizo, Artigas \& Banterla, 2011; Kamil \& Chen, 2001; Ekstrom \& Yonelinas, 2020). In addition, stable environmental cues may also improve the understanding of the perspective shifts. For example, participants can use the change in the egocentric relations to those cues as well as the changes in the visual projection of those cues to understand how their position in space has changed following a perspective shift. Thus, we propose that enriching the environment with further spatial information will reduce the uncertainty about the object position after the perspective shift. If our conjecture about the role of uncertainty is correct, then reducing uncertainty by adding cues in the environment should reduce the perspective shift related bias by decreasing the weight given to the egocentric anchor as well as by improving the adjustment process.

It is possible that the uncertainty about the perspective shift may have also arisen from the way we introduced perspective shifts. For example, in our previous studies, the perspective shift consisted of both translation and rotation (Segen et al., 2021c, 2021d). Specifically, the camera moved on a circle such that it translated to one direction and at the same time rotated in the opposite direction. This combination of camera translation and rotation is typical for spatial perspective-taking tasks (Montefinese et al., 2015; Muffato et al., 2019; Hilton et al., 2020; Segen et al., 2021a, 2021b; Sulpizio et al., 2013; Schmidt et al., 2007) as it ensures that the same part of the environment is visible before and after the perspective shift. Given the small perspective shifts introduced in our studies (that involved small translations requiring only 20 to 30 -degree rotations), the resulting images looked quite similar. This may have produced difficulties in understanding the perspective shifts, increasing participants' uncertainty regarding their movement within the environment. For example, if 
participants thought that the camera movement between encoding and test was smaller than it was, this could have caused a bias that is congruent with the direction of the perspective shift.

So far, the unique role of camera rotations and of translations during perspective taking has not been studied. Although our previous research suggests that the observed perspective shift related bias is linked to the introduction of camera movements during perspective shifts, it is not clear whether it is driven by camera translations or rotations separately or by the specific combination of the two. Therefore, the main aim of this study is to investigate the contribution of camera rotations and translations to the perspective shift related bias that we have observed in our previous studies (Segen et al., 2021c, 2021d).

Although no perspective-taking studies have investigated the role of translations and rotations separately, this has been done in tasks assessing spatial updating based on real or imagined body movements (Rieser, 1989, Wraga, 2003; Presson \& Montello, 1994; Easton \& Sholl, 1995). In such tasks, participants memorize an array of objects and are then either asked to move or to imagine moving to a different position in the array and point to one of the objects from that new position. Results show that, with physical movement, no differences in performance are present when the new position is reached by translation or rotation. However, when participants are asked to imagine moving to the new position, rotations lead to greater errors and longer response times than translations (Rieser, 1989; Presson \& Montello, 1994; Sancaktar \& Demirkan, 2008; Easton \& Sholl, 1995). The difficulties in imagined rotations are also highlighted by difficulties in using maps that are misaligned with participants' orientation in space (Levine, Jankovic, \& Palij, 1982; Presson \& Hazelrigg, 1984; Roskos-Ewoldsen, McNamara, Shelton, \& Carr, 1998). It is, however, not clear whether or how these results translate to spatial perspective taking tasks, as participants do not need to "imagine" rotations or translations, instead they need to use the available information to 
determine how they have moved in space. The current study aims to shed some light on this issue.

Another aim of the current study is to investigate potential ageing differences in object location memory. Previous research suggests that ageing is associated with difficulties in memorising and recognising object locations across different perspectives (Hartley et al., 2007; Montefinese, et al., 2015; Muffato, et al. 2019; Hilton et al., 2020; Segen et al., 2021a) with some studies reporting a specific deficit in spatial perspective taking abilities (e.g. Watanabe, 2011; Montefinese, et al., 2015; Segen et al., 2021a). Furthermore, there is evidence for agerelated declines in the precision of encoding spatial locations. For example, in a number of studies, older adults were found to be less precise in estimating previous locations of objects presented on a computer screen compared to younger adults, despite positioning the objects in the correct region of the stimuli (Pertzov, Heider, Liang, \& Husain, 2015; Nilakantan, Bridge, VanHaerents, \& Voss, 2018). Additionally, there are report of age-related declines in precision of spatial memory in a virtual Morris water maze task (McAvan et al., 2021). In this study, participants physically navigated in a virtual environment presented via a head mounted display. Interestingly, older participants showed comparable performance for memory of object locations across different perspectives and displayed similar strategy use, yet their memory of object locations was less precise. These age-related declines in the precision of spatial representations may be caused by differential age-related changes in the anterior and posterior hippocampus. Indeed, a recent longitudinal study (Langnes et al., 2019) reported that the posterior hippocampus, typically associated with fine-grained spatial processing, was more affected by ageing than the anterior hippocampus, which is involved in the formation of coarser spatial representations (Røe Evensmoen et al., 2013; Nadel, Hoscheidt \& Ryan, 2013).

\subsubsection{Aims \& Hypotheses}


In the current study we present two experiments. The key aim of Experiment 1 is to provide a conceptual replication of Segen et al., (2021d) in which we found a perspective shift related bias during object position estimates. In the original task (Segen et al., 2021d), participants memorised the position of a target object that was always located on a plank in a virtual room. Then, following a short delay and a perspective shift, the target object disappeared, and participants were asked to indicate its position by selecting one of several predefined positions. In the current study, we introduced two key changes compared to the original task. First, we have removed the plank which may have acted as an influential cue that restricted the possible locations where the target object could be placed. Second, instead of presenting participants with predefined object positions that were overlaid on the plank during the test phase, participants' responses were unconstrained, and they could indicate the position of the target object anywhere in the environment. Removing the plank and the positional markers reduced the risk that participants relied on strategies we did not control for and which could be responsible for the perspective shift related bias.

The key aim of Experiment 2 was to investigate the contribution of camera rotations and translations to the perspective shift related bias. To do so, we manipulated camera rotation and the translations independently during the perspective shifts. We also investigated if enriching the environment by including additional objects that could be used as cues would improve participants' ability to remember the precise positions of the target object across different camera movements (rotations and translations). Furthermore, we examined the role aging has on the precision with which participants estimate target object positions across different camera movements. Lastly, we investigated if older adults are differentially affected by camera rotations, translations, and the presence of additional cues in the environment compared to younger adults. 
We postulate that the presence of the additional objects in the environment should improve the precision of participants' representations of the target object location (Cánovas et al, 2011; Chamizo et al., 2011; Kamil \& Chen, 2001; Ekstrom \& Yonelinas, 2020) as well as the understanding of the perspective shifts. Thus, we predicted smaller errors and a reduced perspective shift related bias when additional cues are present. Given the age-related declines in spatial memory (Hartley et al., 2007; Montefinese, et al., 2015; Muffato, et al. 2019; Hilton et al., 2020; Segen et al., 2021a, 2021b) and precision of spatial encoding across 2D stimuli (Pertzov et al. 2015; Nilakantan et al., 2018) together with possible perspective-taking deficits (Watanabe, 2011; Montefinese, et al., 2015; Segen et al., 2021b), we predicted that older adults would be less precise compared to younger adults and would display a larger bias related to camera movements in the environment. This prediction is based on our previous research that showed that older adults were more affected than younger adults by the direction of the perspective shift when estimating the direction in which the object has moved (Segen et al, 2021a). To our knowledge, this is the first study using spatial perspective-taking in which camera rotations and translations are decoupled. We therefore have no specific prediction on how the camera movements would contribute to performance and the perspective shift related bias. It is possible that participants will be more affected by camera rotations, as previous research on spatial updating shows that imagined rotations are harder than imagined translations (Rieser, 1989; Presson \& Montello, 1994; Sancaktar \& Demirkan, 2008; Easton \& Sholl, 1995). Alternatively, if the perspective shift related bias that we reported in earlier studies was driven by the specific camera movements that we have used where the rotation is always in a different direction to the translation, we would expect the bias to be present only in such situations

\subsection{Experiment 1}

\subsubsection{Introduction}


In Experiment 1 we introduced a modified version of a task we used in Segen et al., (2021d) to investigate spatial memory across different perspectives. In this task participants memorised scenes containing a target object and then, following a short delay, they were presented with a second image showing the same scene from a different perspective but without the target object. When viewing the second scene, participants had to indicate the position of the target object.

The main aim of this experiment $k$ was to provide a conceptual replication of the results reported in Segen et al. (2021d). Thus, we predict that participants' errors will be biased in the direction of the perspective shift.

\subsubsection{Method}

\subsubsection{Participants}

Twenty-eight participants aged between 18 to 35 years of age (mean age $=24.04$ years, $\mathrm{SD}=4.69 ;$ age range $=18-33$ years; 16 females and 12 males) took part in this study. Participants were recruited through the participant recruitment system of Bournemouth University and received course credit for their participation. All participants gave their informed consent in accordance with the Declaration of Helsinki (World Medical Association, 2013).

\subsubsection{Materials}

The virtual environment was designed with 3DS Max 2018 (Autodesk) and consisted of a square $9.8 \mathrm{~m} \times 9.8 \mathrm{~m}$ room. Posters depicting famous landmarks were placed on the walls of the virtual room. The landmarks were chosen based on familiarity ratings obtained from previous research by Hamburger \& Roser, (2014). The target object, a potted plant, was placed in one of 18 predefined positions and the scene for encoding was rendered from one of three camera positions (camera locations depicted in Figure 7.1). At test, the object was 
removed, and the scene was rendered from one of the six test camera positions (Figure 7.1) such that the camera either moved to the left or to the right of the encoding position. The experimental stimuli were renderings of the environment with a $58^{\circ}$ horizontal field of view (FOV). A custom asymmetric viewing frustum that resembles natural vision with a $15 \%$ shift in the vertical FOV was used. This asymmetric viewing frustum resembles natural vision and has been found to improve distance perception in virtual environments (Franz, 2005).

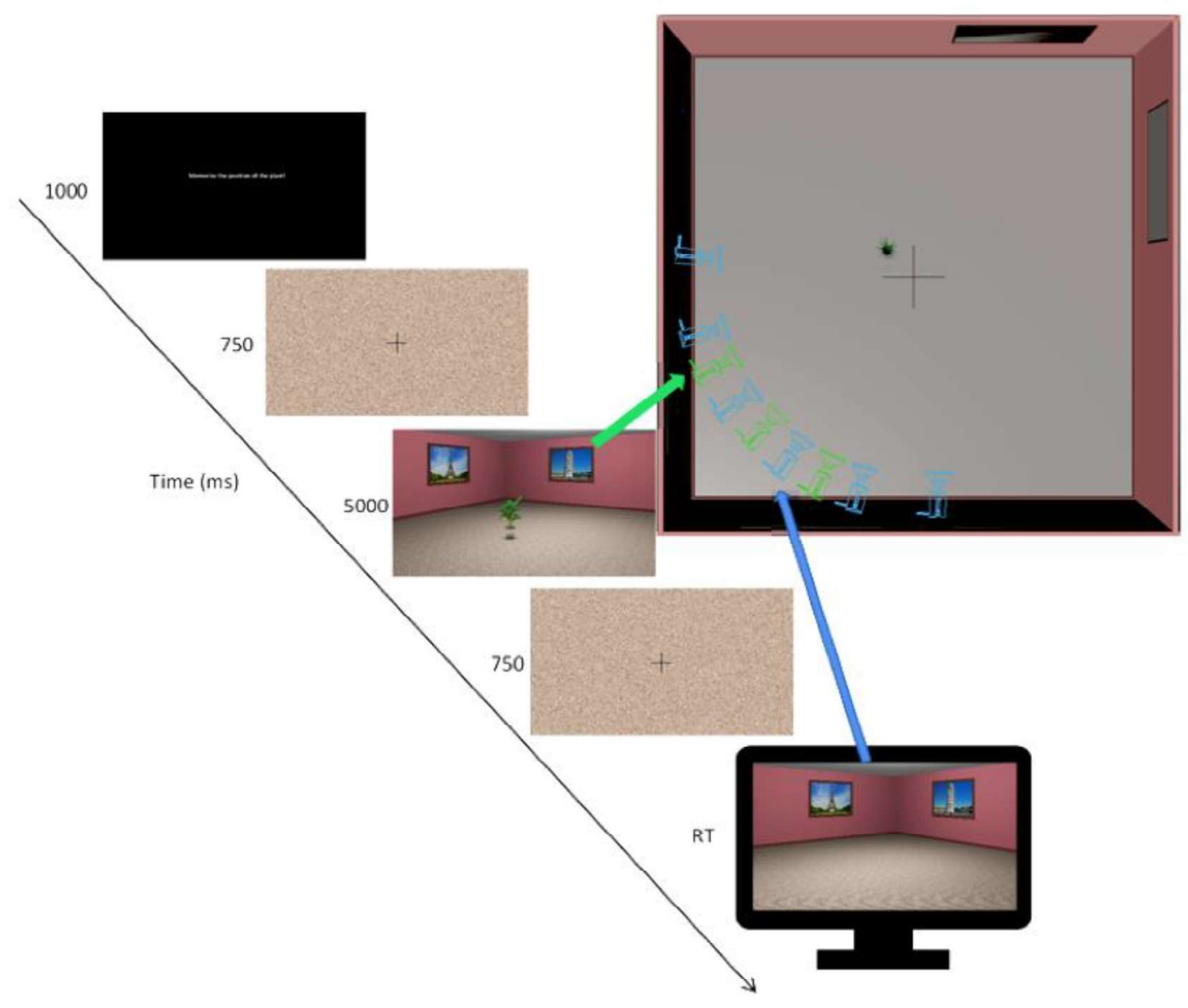

Figure 7.1 A top-down schematic of the virtual environment used in the experiment with camera positions. Green cameras represent camera positions at encoding and blue cameras represent the corresponding camera positions at test. B: Trial structure with green and blue arrows showing the encoding and test cameras used to render the encoding and test scenes 


\subsubsection{Procedure}

The experiment was carried out online using Testable (testable.org). At the beginning of the experiment, participants were asked to adjust the screen zoom settings to ensure that the entire scene was visible during the experiment which was run in full-screen mode. Each experimental trial started with instructions to remember the position of the object (1000ms), this was followed by a fixation cross and a scrambled stimuli mask presented for $750 \mathrm{msec}$ (Figure 1B). In the encoding phase, participants were presented with a rendering of the room with one of the 18 possible target object positions from one of three encoding camera positions for 5 seconds. This was followed by the presentation of a fixation cross and a scrambled stimuli mask for 750 msec. Finally, in the test phase, participants were presented with a rendering of the room without the target object from one of the six possible camera positions (Figure 7.1A). Participants had to indicate the position of the object taking into account the camera movements between encoding and test. Participants moved the mouse cursor to the position where they thought the object was during encoding and clicked to register their responses. They were instructed to use the base of the target object to remember the position it occupied on the floor.

Each of the 18 possible target object positions was presented twice for each of the three encoding camera positions which resulted in 108 experimental trials that took around 25 minutes to complete.

\subsubsection{Results}

Since the main aim of the experiment is to investigate biases in the direction in which participants estimate object locations, only angular errors (i.e., the unsigned distance between the correct object location and participant's response) are reported, with distance errors (Euclidean distance between participants' estimate of the object's position and the object's actual position) presented noted in the supplementary materials. 
To investigate if the direction of the perspective shift between encoding and test biased the direction of participants' position estimates for the object, we focused on signed angular error (Figure 7.2). Positive and negative errors indicate that the object was estimated to be to the right or the left (respectively) of the correct object position. We ran linear mixed-effects models (LME) using LME4 (Bates et al. 2015) in R (R Core Team, 2013) to investigate the role the Perspective Shift Direction (PSD) had on participants signed angular errors. PSD (Left/Right) was coded using sum contrasts such that left perspective shifts were compared to the average errors for the Left and Right PSD. We found that PSD (Left) influenced participants' errors ( $\beta=$ 6.712, $\mathrm{SE}=0.426, \mathrm{t}=-15.743)$, with participants positioning the target object further to the left when the perspective shift was to the Left. If we reverse the contrasts such that Right PSD is compared to the grand average, a reverse pattern is found with participants' errors shifted to the right for Right PSD. In other words, participants exhibited a bias in their estimates that were in the same direction as that of the perspective shift between encoding and test (Figure 7.2).

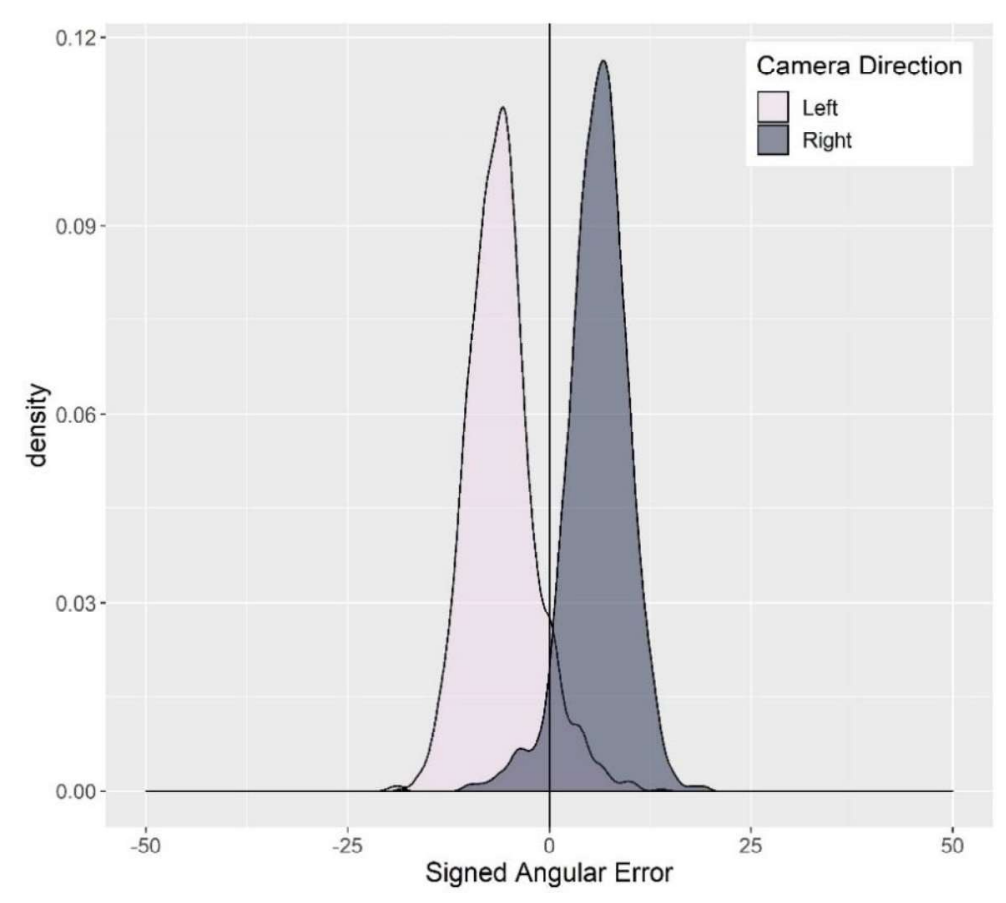

Figure 7.2 Distribution of Signed Angular Errors as a function of Camera Direction 


\subsubsection{Discussion}

Experiment 1 showed that when indicating target object positions, participants systematically made errors in the same direction as the perspective shift. We also found that participants systematically overestimated the distance of the object as they had placed the object further than its actual position. The presence of a systematic shift in participants' estimates of the position of the target object in the same direction as the perspective shift provides a conceptual replication of our previous findings (Segen et al., 2021d). Notably, in the original task, the objects were always placed on a plank and participants were provided with a set of predefined positional markers on the plank and indicated the position of the target object by selecting one of the markers. In the current task, we removed both the plank and the positional markers to rule out the possibility that these cues were related to the perspective shift induced bias. Thus, the presence of a systematic influence of the perspective shift on participants' object location estimates in the current study suggests that the bias is more likely to be driven by camera movements in the environment. In Experiment 2, we further explore what may be driving the perspective shift induced bias.

\subsection{Experiment 2}

\subsubsection{Introduction}

It is possible that the camera movements used in Experiment 1 and in other studies with spatial perspective tasks (Montefinese et al., 2015; Muffato et al., 2019; Hilton et al., 2020; Segen et al., 2021a, 2021b ,2021c, 2021d; Sulpizio et al., 2013) contributed to the perspective shift related bias in target object position estimates. Specifically, we speculated that there might be something special about this combination of camera rotations and translations, where the camera translates in one direction and rotates in the opposite direction, that gives rise to the perspective shift related bias. For example, participants may have difficulties in correctly perceiving the size of the perspective shift since the images 
rendered from both perspectives look strikingly similar. This is because the rotation in the opposite direction to the translation ensured that the same features of the scene remained visible. This could lead participants to systematically underestimate the extent of the camera movement and lead to the systematic shift in the errors in direction of the camera shift.

The key aim of Experiment 2 was, therefore, to investigate the contribution of camera rotations and translations to the perspective shift related bias. To do so, we varied camera rotations and translations independently by creating situations with rotations but without translations and vice versa. In addition, we introduced camera movements that we and others have used in previous work (Segen et al., 2021a, 2021b ,2021c, 2021d; Montefinese et al, 2015; Muffato et al., 2019; Hilton et al., 2020; Sulpizio et al., 2013; Schmidt et al., 2007), in which the camera translates and rotates in opposite directions, to investigate if only this specific combination of camera movements gives rise to the perspective shift related bias. Lastly, we added a situation where the camera translates and rotates in the same direction.

We have argued that uncertainty about the location of the target object following perspective shift is likely to contribute to the perspective shift related bias (Segen et al., 2021c, 2021d). We expect that enriching the environment with additional stable environmental cues will help participants to better estimate the exact object location (Cánovas et al., 2011, Chamizo et al., 2011) and to understand the perspective shift, thereby reducing the uncertainty regarding the target position. The second aim of Experiment 2 was, therefore, to investigate if the introduction of stable environmental cues (two round pillars) would improve overall precision and reduce the effect of the perspective shift on participants' performance.

In addition, in Experiment 2 we investigated whether ageing mediates the effect of camera translations and rotations, as well as the effect that the additional cues may have on the ability to precisely encode and retrieve object positions and, specifically, on the perspective shift related bias. Since ageing is associated with declines in the precision of 
spatial memory (Pertzov et al. 2015; Nilakantan et al., 2018; Segen et al., 2021a, 2021c;

McAvan et a., 2021) and has been linked with perspective taking deficits (Watanabe, 2011;

Montefinese et al., 2015; Segen et al., 2021a), we expected that older adults would be less

precise in estimating the position of the target object. Due to reduced precision, we expect

that older adults would experience greater uncertainty about the exact position of the target

object and therefore show a more pronounced error bias in positioning the target object in the direction of the perspective shift.

\subsubsection{Method}

\subsubsection{Participants}

Forty-five young adults (mean age $=20.70$ years, $S D=3.26$; age range $=18-33$ years; 25 females and 20 males) and forty-one older adults aged 60 years and over (mean age=68.00, $S D=6.44$, age range $=60-86 ; 21$ females and 20 males) took part in this study. Participants were recruited either through the participant recruitment system of Bournemouth University or Prolific (https://www.prolific.co), an online participant recruitment system. Older adults received monetary compensation for their time whilst younger participants received course credit. All participants gave their written informed consent in accordance with the Declaration of Helsinki (World Medical Association, 2013).

\subsubsection{Design}

The experiment followed a mixed 2 (Age Group: Young/Older) × 2 (Environment: No Columns/Additional Columns) $\times 3$ (Camera Translation: Left Translation/No Translation/Right Translation) x 3 (Camera Rotation: Left Rotation/No Rotation/Right Rotation) design with Environment, Camera Translation and Camera Rotation manipulated within participants and Age Group manipulated between participants. 


\subsubsection{Materials}

We used the same virtual environment as in Experiment 1. In this experiment, however, we only used 4 predefined target object positions and the encoding scenes were rendered only from the central camera position (Figure 7.1A). During encoding, the camera was oriented to always face the centre of the room. For the test stimuli, the target object was removed and the scenes were rendered from one of the three test camera positions such that the camera either remained in the same position, moved to the left, or moved to the right by $1 \mathrm{~m}$ from the encoding position. The rotation of the camera was also manipulated at test such that the camera rotated by $10^{\circ}$ to the left, $10^{\circ}$ to the right, or did not rotate. This design yielded a total of nine possible combinations of camera position and rotation for the test stimuli (examples of stimuli shown in Figure 7.3A). In the Additional Columns condition, two round columns that differed in colour were added to the environment (Figure 7.3B). 
A
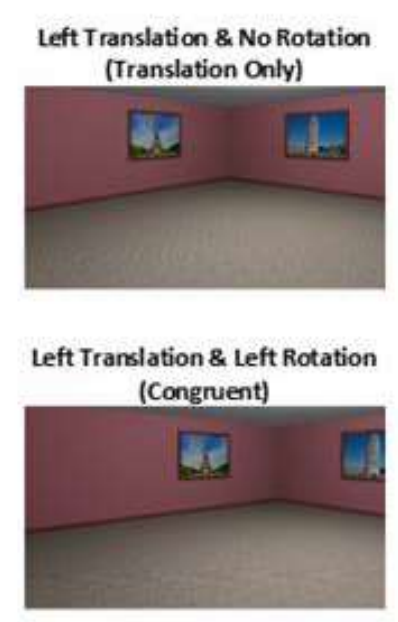

Left Translation \& Right Rotation (Incongruent)

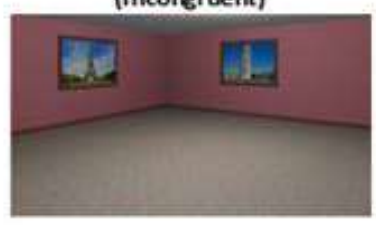

Encoding Stimuli

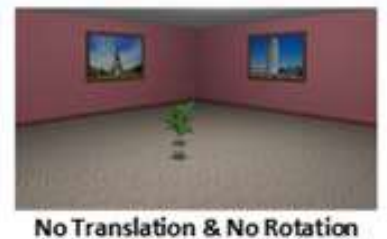

(No Change)

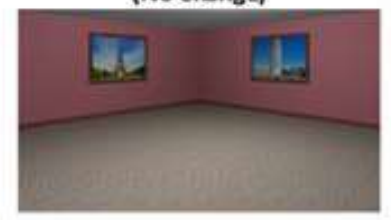

No Translation \& Left Rotation

(Rotation Only)

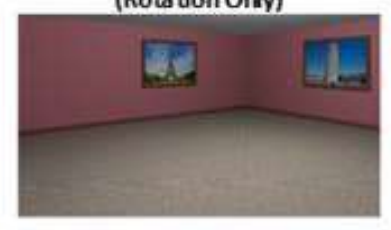

No Translation \& Right Rotation (Rotation Only)

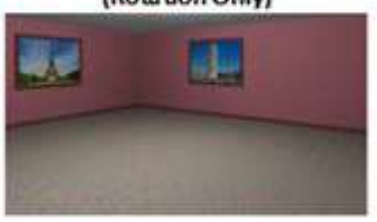

Right Translation \& No Rotation (Translation)

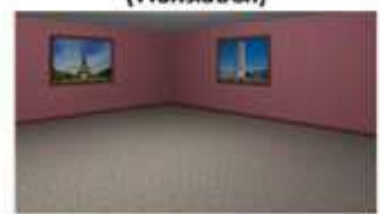

Right Translation \& Left Rotation (Incongruent)

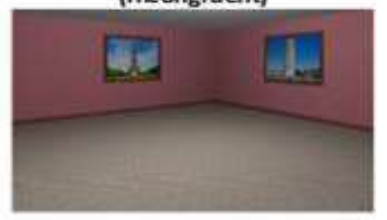

Right Translation \& Right Rotation (Congruent)

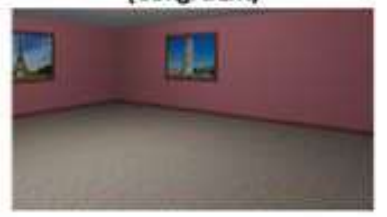

B
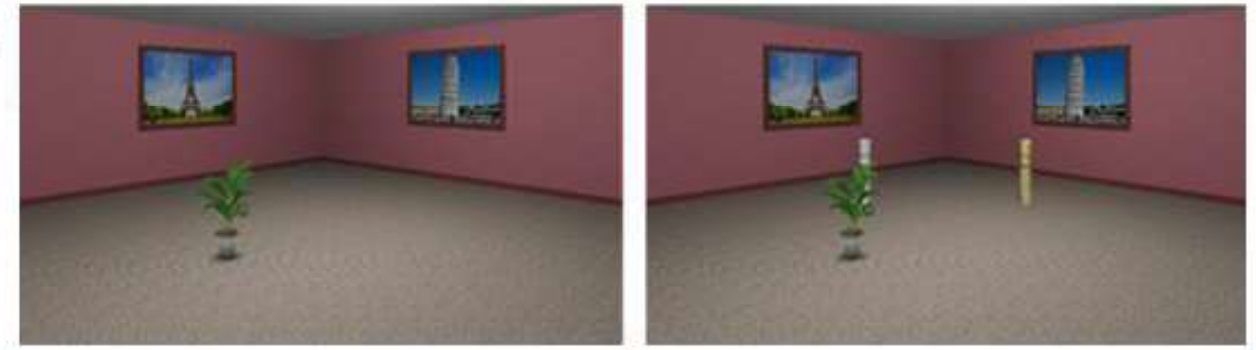

Figure 7.3 Sample scene during encoding. A Test scenes across different camera translation and rotation combinations; B Examples of scenes during encoding, depicting the No Columns and Additional Columns Environment conditions.

\subsubsection{Procedure}

The experimental procedure was identical to that of Experiment 1. Each of the 4 possible target object positions were presented twice for each Camera Translation, Camera Rotation and Environment combination. This resulted in a total of 144 experimental trials that 
were preceded by 2 practice trials. The entire study took approximately 30 minutes to complete and was run online using Testable (testable.org).

\subsubsection{Data Analysis}

Data were analysed with LMMs and included Age Group (Young/Older) and Environment (No Columns/Additional Columns) in all the models. Effect coding was used to set contrasts. To reduce the complexity of some of the models we combined Camera Translation and Camera Rotation into a single factor we refer to as Camera Movement (Figure 3B). This resulted in 5 levels, No Movement (No Translation and No Rotation), Rotation Only (No Translation and Left or Right Rotation), Translation Only (No Rotation and Left or Right Translation), Congruent movement (Left Translation and Left Rotation or Right Translation and Right Rotation) and Incongruent movement (Left Translation and Right Rotation or Right Translation and Left Rotation). Camera movement was used to analyse Absolute Angular Errors, contrasts were set using treatment coding with No Movement used as the baseline. In the analysis of Signed Angular Error, Camera Translation and Camera Rotation were used as separate fixed factors and were also coded using treatment coding with No Translation and No Rotation used as a baseline, respectively. All models included a random by-subject and byitem intercept. Prior to analysis, outlier responses were removed using the interquartile range method on individual absolute distance error $(\mathrm{m})$ distributions, which led to a $3.6 \%$ data loss.

\subsubsection{Results}

\subsubsection{Absolute angular errors}

The LMM analysis showed that the absolute angular errors were larger with camera movements than without (Table 7.1 and Figure 7.4). Specifically, there was a small increase in angular errors when camera rotations were introduced (Rotation Only trials), a larger increase in errors was found for Incongruent trials, followed by an even larger increase for Translation Only trials and for Congruent trials. We also found a significant interaction between 
Environment and Camera Direction, with a lower increase of error in the Additional Objects condition with the introduction of Congruent and Translation Only trials. In addition, we also found an interaction between Camera Movement and Age Group with a larger increase of error in the Incongruent and Translation Only trials in Older Adults.

Table 7. 1 Coefficients from Absolute Angular Error LME analysis

\begin{tabular}{|c|c|c|c|}
\hline \multirow[b]{2}{*}{ Predictors } & \multicolumn{3}{|c|}{ Absolute Angular Error } \\
\hline & Estimates & $\begin{array}{l}\text { std. } \\
\text { Error }\end{array}$ & t-value \\
\hline (Intercept) & 1.718 & 0.147 & 11.659 \\
\hline Environment (Additional Columns) & -0.137 & 0.126 & -1.084 \\
\hline Camera Movement (Congruent) & 3.373 & 0.155 & 21.753 \\
\hline Camera Movement (Incongruent) & 2.024 & 0.155 & 13.079 \\
\hline Camera Movement (Rotation Only) & 0.770 & 0.155 & 4.975 \\
\hline Camera Movement (Translation Only) & 2.721 & 0.155 & 17.581 \\
\hline Age Group (Older) & 0.033 & 0.104 & 0.318 \\
\hline $\begin{array}{l}\text { Environment (Additional Columns)*Camera Movement } \\
\text { (Congruent) }\end{array}$ & -0.563 & 0.155 & -3.633 \\
\hline $\begin{array}{l}\text { Environment (Additional Columns)*Camera Movement } \\
\text { (Incongruent) }\end{array}$ & -0.258 & 0.155 & -1.666 \\
\hline $\begin{array}{l}\text { Environment (Additional Columns)*Camera Movement } \\
\text { (Rotation Only) }\end{array}$ & -0.259 & 0.155 & -1.676 \\
\hline $\begin{array}{l}\text { Environment (Additional Columns)*Camera Movement } \\
\text { (Translation Only) }\end{array}$ & -0.541 & 0.155 & -3.494 \\
\hline Environment (Additional Columns)*Age Group (Older) & 0.049 & 0.071 & 0.694 \\
\hline
\end{tabular}




\begin{tabular}{|c|c|c|c|}
\hline Camera Movement (Congruent)*Age Group (Older) & 0.087 & 0.088 & 0.986 \\
\hline Camera Movement (Incongruent)*Age Group (Older) & 0.263 & 0.087 & 3.005 \\
\hline Camera Movement (Rotation Only)*Age Group (Older) & -0.090 & 0.087 & -1.030 \\
\hline Camera Movement (Translation Only)*Age Group (Older) & 0.195 & 0.087 & 2.225 \\
\hline $\begin{array}{l}\text { Environment (Additional Columns)*Camera Movement } \\
\text { (Congruent) *Age Group (Older) }\end{array}$ & -0.045 & 0.088 & -0.510 \\
\hline $\begin{array}{l}\text { Environment (Additional Columns)*Camera Movement } \\
\text { (Incongruent) *Age Group (Older) }\end{array}$ & -0.132 & 0.087 & -1.507 \\
\hline $\begin{array}{l}\text { Environment (Additional Columns)*Camera Movement } \\
\text { (Rotation Only) * Age Group (Older) }\end{array}$ & -0.078 & 0.087 & -0.893 \\
\hline $\begin{array}{l}\text { Environment (Additional Columns)*Camera Movement } \\
\text { (Translation Only)*Age Group (Older) }\end{array}$ & -0.091 & 0.087 & -1.040 \\
\hline
\end{tabular}

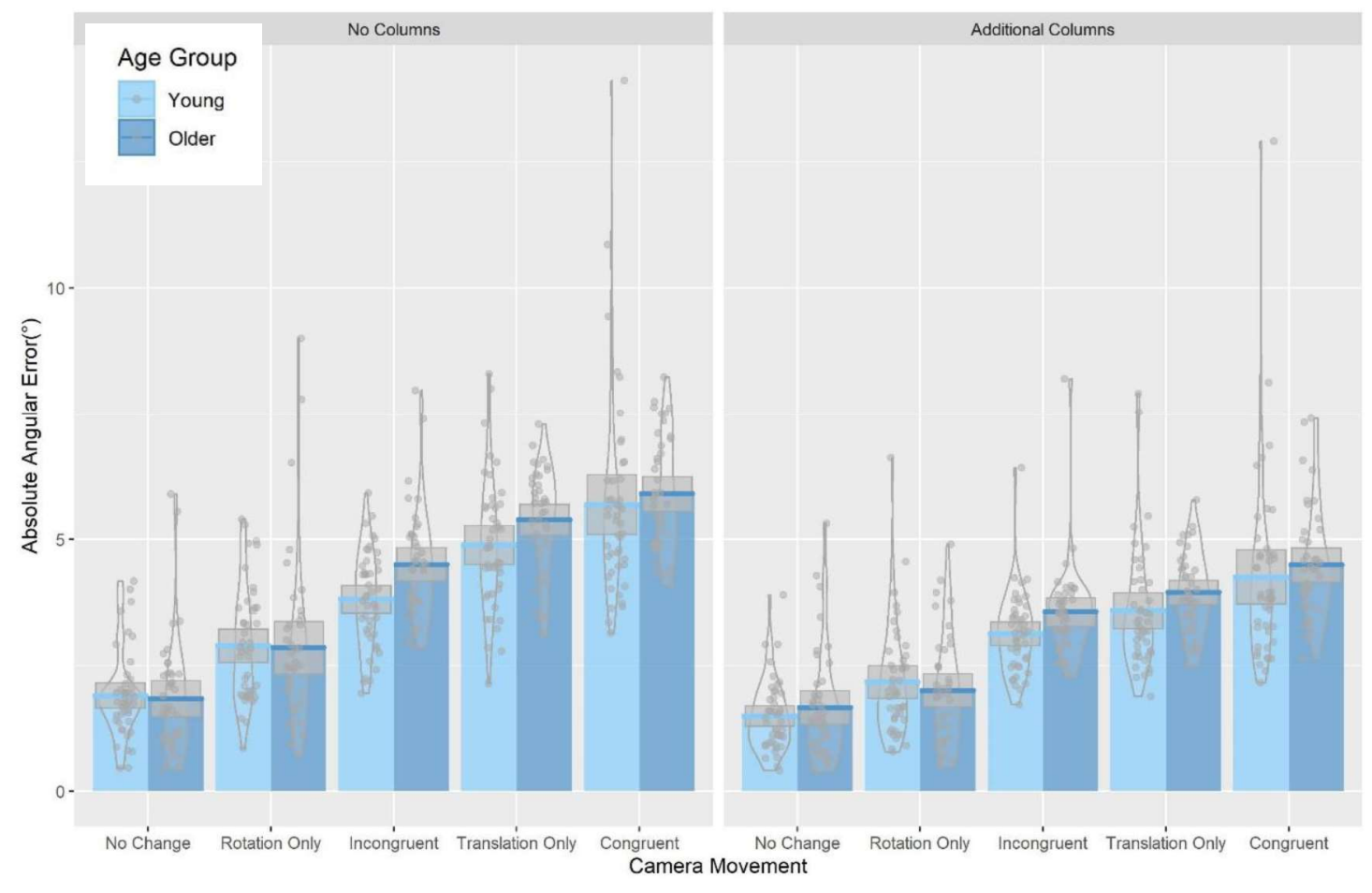

Figure 7.4 Absolute angular error as a function of Camera Movement, Environment and Age Group 


\subsubsection{Signed Angular Errors}

To investigate which camera movements systematically bias the direction of object location estimates, we focused on signed angular error. Positive errors indicate that the target object was estimated to the right of the correct position and negative errors indicate errors to the left of the correct position. In this model we have included Camera Translations and Camera Rotations as separate fixed factors, as otherwise the errors for different directions of camera rotations and translations could cancel each other out.

The LMM analysis (see Table 7.2 and Figure 7.5) showed that Camera Rotations introduced a small bias in errors in the direction of the rotation. Camera Translations had a much larger effect on participants' signed angular errors, with participants' estimates of target object locations showing a large shift in the direction of the translation. We also found an Environment by Camera Translation interaction: errors were less biased when the camera translated to the left in the Additional Columns condition than in the No Columns condition. A similar trend ( $\mathrm{t}=1.955)$ was also present when the camera translated to the right.

We also found an interaction between Camera Rotation and Age Group, with older adults showing smaller error bias when camera rotations were present compared to younger adults. This effect was only significant for rotations to the left, but the numerical trend is present also for rotations to the right. In contrast, older adults seem to be more affected than young adults by camera translations. This was corroborated by the presence of an Age Group by Camera Translation interaction with older adults showing a greater error bias in the direction of camera translations compared to young adults. Again, the interaction was only significant for camera translations to the right with a similar trend for camera translation to the left.

To quantify the differences between the effect of camera rotations and translations, we conducted linear hypothesis tests and found that the effect for each direction of the 
camera rotation were significantly different for the corresponding effect for each direction of camera translation, i.e. left translation vs left rotations $(p<.001)$. Next, we compared the magnitude of that difference and found that the effect of camera translation on signed angular error is almost threefold (2.85) to that of camera rotations $(p<.05)$.

Table 7.2 Coefficients Signed Absolute Angular Error LME analysis

\begin{tabular}{|c|c|c|c|}
\hline \multirow[b]{2}{*}{ Predictors } & \multicolumn{3}{|c|}{ Signed Angular Error } \\
\hline & Estimates & $\begin{array}{l}\text { std. } \\
\text { Error }\end{array}$ & t-value \\
\hline (Intercept) & -0.211 & 0.193 & -1.095 \\
\hline Environment (Additional Columns) & -0.117 & 0.181 & -0.644 \\
\hline Rotation (Left) & -0.631 & 0.257 & -2.459 \\
\hline Rotation (Right) & 0.765 & 0.257 & 2.977 \\
\hline Translation (Left) & -3.647 & 0.257 & -14.199 \\
\hline Translation (Right) & 4.036 & 0.257 & 15.728 \\
\hline Age Group (Older) & -0.185 & 0.115 & -1.607 \\
\hline Environment (Additional Columns)*Rotation (Left) & 0.128 & 0.257 & 0.498 \\
\hline Environment (Additional Columns)*Rotation (Right) & -0.044 & 0.257 & -0.171 \\
\hline Environment (Additional Columns)*Translation (Left) & 0.502 & 0.257 & 1.955 \\
\hline Environment (Additional Columns)*Translation (Right) & -0.625 & 0.257 & -2.435 \\
\hline Rotation (Left)*Translation (Left) & 0.054 & 0.363 & 0.148 \\
\hline Rotation (Right)*Translation (Left) & 0.192 & 0.363 & 0.530 \\
\hline Rotation (Left)*Translation (Right) & -0.360 & 0.363 & -0.990 \\
\hline
\end{tabular}




\begin{tabular}{|c|c|c|c|}
\hline Rotation (Right)*Translation (Right) & 0.042 & 0.364 & 0.116 \\
\hline Environment (Additional Columns)*Age Group (Older) & 0.039 & 0.094 & 0.408 \\
\hline Rotation (Left)*Age Group (Older) & 0.332 & 0.134 & 2.487 \\
\hline Rotation (Right)*Age Group (Older) & -0.207 & 0.134 & -1.545 \\
\hline Translation (Left)*Age Group (Older) & -0.170 & 0.134 & -1.266 \\
\hline Translation (Right)*Age Group (Older) & 0.273 & 0.134 & 2.041 \\
\hline $\begin{array}{l}\text { Environment (Additional Columns)*Rotation } \\
\text { (Left)*Translation (Left) }\end{array}$ & 0.148 & 0.363 & 0.408 \\
\hline $\begin{array}{l}\text { Environment (Additional Columns)*Rotation } \\
\text { (Right)*Translation (Left) }\end{array}$ & -0.113 & 0.363 & -0.312 \\
\hline $\begin{array}{l}\text { Environment (Additional Columns)*Rotation } \\
\text { (Left)*Translation (Right) }\end{array}$ & 0.173 & 0.363 & 0.476 \\
\hline $\begin{array}{l}\text { Environment (Additional Columns)*Rotation } \\
\text { (Right)*Translation (Right) }\end{array}$ & 0.090 & 0.364 & 0.246 \\
\hline $\begin{array}{l}\text { Environment (Additional Columns)*Rotation (Left)*Age } \\
\text { Group (Older) }\end{array}$ & -0.021 & 0.134 & -0.154 \\
\hline $\begin{array}{l}\text { Environment (Additional Columns)*Rotation (Right)*Age } \\
\text { Group (Older) }\end{array}$ & -0.017 & 0.134 & -0.127 \\
\hline $\begin{array}{l}\text { Environment (Additional Columns)*Translation (Left)*Age } \\
\text { Group (Older) }\end{array}$ & -0.005 & 0.134 & -0.035 \\
\hline $\begin{array}{l}\text { Environment (Additional Columns)*Translation (Right)*Age } \\
\text { Group (Older) }\end{array}$ & -0.121 & 0.134 & -0.906 \\
\hline Rotation (Left)*Translation (Left)*Age Group (Older) & -0.116 & 0.190 & -0.610 \\
\hline Rotation (Right)*Translation (Left)*Age Group (Older) & -0.013 & 0.190 & -0.066 \\
\hline Rotation (Left)*Translation (Right)*Age Group (Older) & -0.169 & 0.189 & -0.892 \\
\hline Rotation (Right)*Translation (Right)*Age Group (Older) & 0.153 & 0.191 & 0.804 \\
\hline $\begin{array}{l}\text { Environment (Additional Columns)*Rotation } \\
\text { (Left)*Translation Left)* Age Group (Older) }\end{array}$ & -0.156 & 0.190 & -0.822 \\
\hline $\begin{array}{l}\text { Environment (Additional Columns)*Rotation } \\
\text { (Right)*Translation (Left)*Age Group (Older) }\end{array}$ & 0.108 & 0.190 & 0.570 \\
\hline $\begin{array}{l}\text { Environment (Additional Columns)*Rotation } \\
\text { (Left)*Translation (Right)* Age Group (Older) }\end{array}$ & -0.030 & 0.189 & -0.157 \\
\hline
\end{tabular}



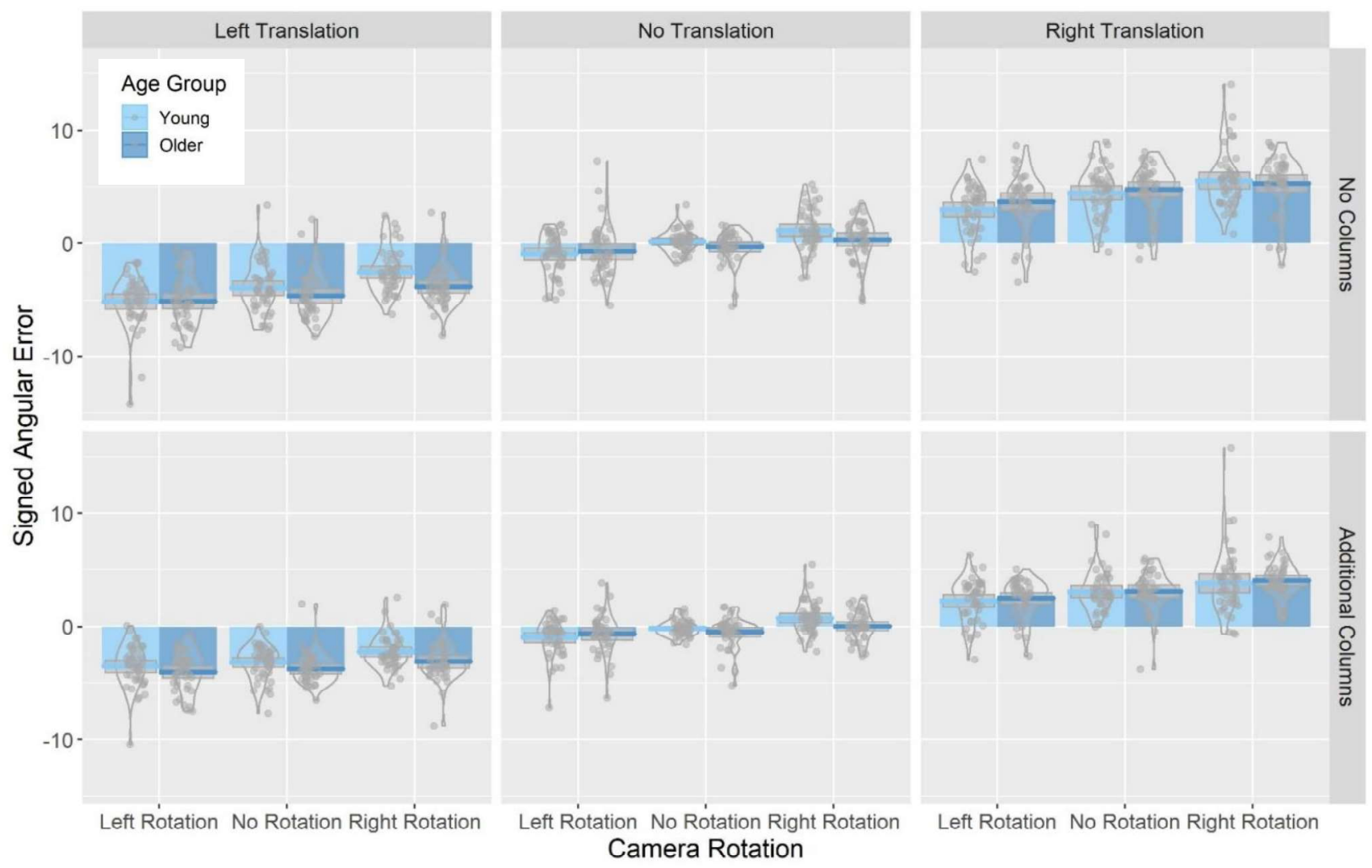

Figure 7.5 Signed angular error as a function of Camera Translations, Camera Rotations and Age Group in the No Columns condition (top panel) and Additional Columns (bottom panel)

\subsubsection{Linear combination of errors for camera rotations and translations}

To investigate how camera rotations and translations interact, we modelled predictions for combined movements based on rotation and translation data. Specifically, we created three models (Figure 7.6), one in which signed errors were solely affected by camera rotation (Rotation Only model), one in which signed errors were solely affected by camera translation (Translation Only model) and one which assumed an additive influence of camera rotation and translation (Additive Model). The predictions of the three models, along with the experimental 
data, are presented in Figure 8. It is apparent that participants' errors are unlikely to be driven solely by camera rotations, whilst both the Translation Only model and the Additive Model fit the experimental data well. However, the Additive Model provides a significantly better fit than the Translation Only model (Translation Only RSS=1677.5, Additive Model RSS=1146.1, $F=35748, p<.001)$. The close fit of the predictions of the additive model for the combined camera movements with the actual data suggests that camera rotation and camera translation independently influence participants' performance.

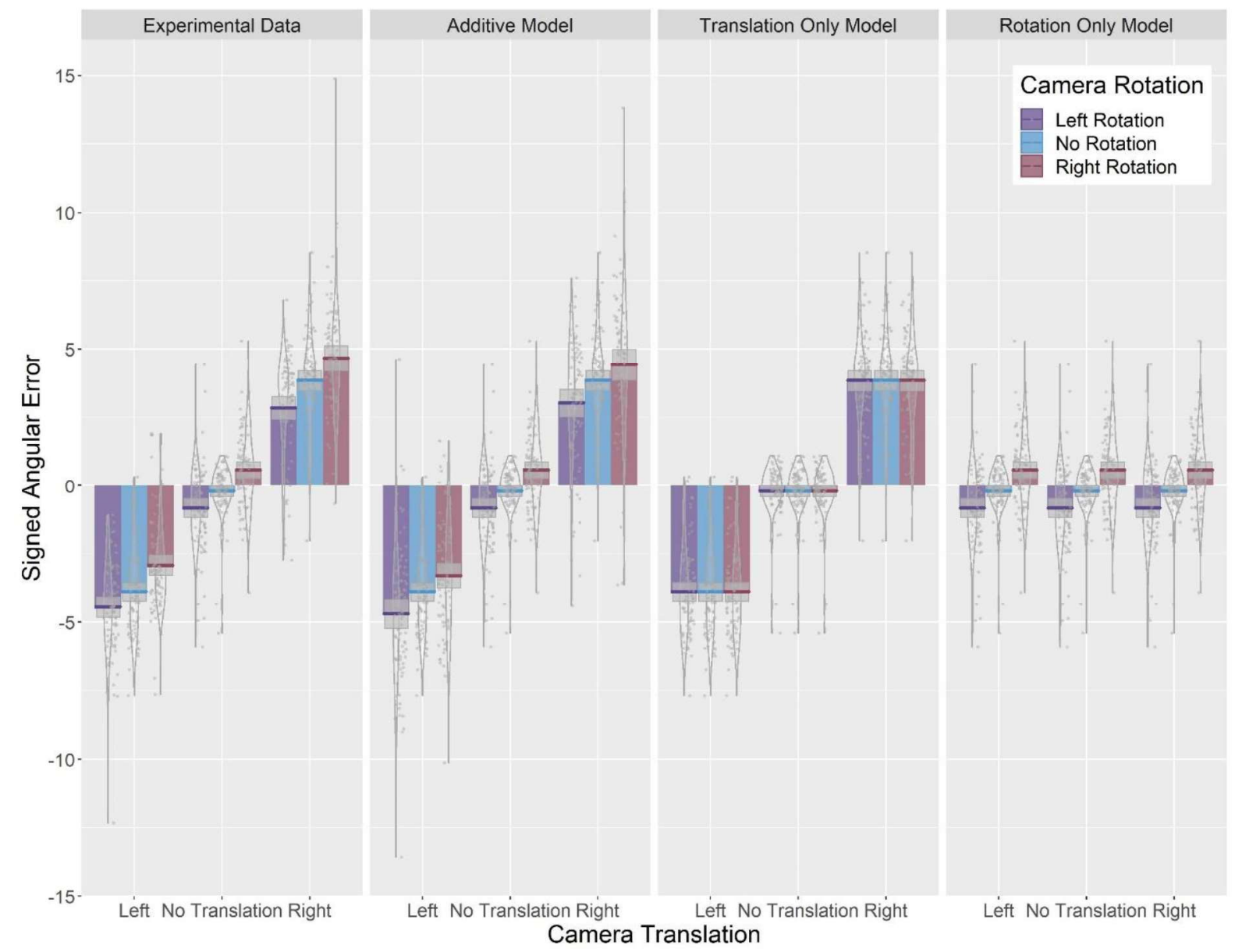

Figure 7.6 Experimental Data and predictions of the Additive, Translation Only and Rotation Only models 


\subsubsection{Absolute Distance Errors}

Lastly, to investigate differences in the precision with which participants recalled the position of objects following a perspective shift, we focused on the Absolute Distance Errors The LMM analysis (complete results are presented in supplementary materials) showed that errors decreased in the Additional Columns Environment $(\beta=-0.983, \mathrm{SE}=0.013, \mathrm{t}=-6.471$ ). Moreover, the introduction of any camera movements increased participants' error when compared to the No Movement baseline. Notably, the increase was not uniform. The lowest increase in error occurred when camera rotations were introduced (Rotation Only; $\beta=0.034$, $\mathrm{SE}=0.016, \mathrm{t}=2.134$ ), followed by trials with Incongruent camera movements (camera translates and rotates in opposite directions; $\beta=0.074, \mathrm{SE}=0.016, \mathrm{t}=4.707)$. Error increased further in Translation Only trials $(\beta=0.114, \mathrm{SE}=0.016, \mathrm{t}=7.268)$, with the largest errors observed in trials with Congruent camera movements (camera translates and rotates in the same direction; $\beta=0.163, S E=0.016, t=10.298)$. We also found a significant interaction between Environment and Age Group $(\beta=0.020, S E=0.009, t=2.167)$. Error was smaller in the Additional Columns condition than in the No Columns condition, but this difference was larger in younger compared to older adults. This finding suggests that older adults did not benefit from the availability of extra spatial information (extra columns) as much as younger adults did.

\subsubsection{Discussion}

In the present study we investigated the role camera rotations and translations have on the error's participants make when recalling object locations. We also examined if enriching the environment by providing additional spatial information influences the ability to precisely estimate the position of the target object following a perspective shift as well as the perspective shift related bias in the position estimates. We also investigated age-related differences in the precision with which people estimate target object locations. Lastly, we 
examined if older adults are differentially affected by camera rotations and translations as well as by the presence of additional cues when estimating target object locations.

We found that the introduction of any camera movements between encoding and test increased error in estimating the position of the target object. This was the case for both absolute angular deviations and absolute distance errors. Importantly though, the effect of translations was larger than the effect of rotations. Furthermore, we replicated the perspective shift related bias that we described in our previous studies (Segen et al., 2021c, 2021d). Specifically, we found that participants' responses were biased in the direction of camera movements for both rotations and translations, yet this bias was stronger with the introduction of translations.

There were age-related differences in the manifestation of the perspective shift related bias, as older adults were less affected by camera rotations compared to younger adults, whilst at the same time being more affected by camera translations than younger participants. Furthermore, we found that enriching the spatial information in the environment improved the precision with which participants estimated the position of the object following a perspective shift, yet older adults benefited less from the additional spatial information.

In line with our previous research (Segen et al., 2021c), we found that the presence of additional spatial information reduced the systematic bias in participants' object position estimates following a perspective shift, yet this was only true for perspective shifts containing camera translations. Lastly, we showed that a linear additive model of errors for pure rotations and translations described our data well, suggesting that camera rotations and translations affected participants' errors independently.

We attribute the perspective shift related bias to egocentric influences on target object estimates. In the current task there were no self-motion cues that could support the 
automatic updating of egocentric representations of object locations during the perspective shift (Wang \& Spelke, 2002). Instead, spatial perspective taking had to be achieved through more effortful processes (Easton \& Sholl, 1995). Examples of those include using an allocentric representation that contains information of the object-to-object relations (which are independent from own/camera position in the environment) or by engaging in mental transformations of the egocentric representations to ensure that the encoding and test representations align (King et al, 2002; Hegarty \& Waller, 2004). If participants relied solely on an allocentric representation in which the position of the target object was encoded relative to other features in the environment, their own position and movement in the environment should not have influenced their responses and perspective shifts would not have resulted in systematic biases in the same direction as the perspective shift (King et al, 2002; Hegarty \& Waller, 2004). However, if participants relied on egocentric representations, their responses could be biased towards the egocentric estimates derived before the perspective shift (i.e., during encoding) which would result in the systematic shift in the direction of the camera movement.

In the current experiment, we decoupled camera rotations and translations and showed that translations resulted in a substantially larger angular bias in the direction of the camera movement than camera rotations. We propose that the differential effects of camera rotations and translations on participants' performance are driven by differences in how camera rotations and translations affect the egocentric self-to-object relations and on the 2D projections of object-to-object relations. We propose that in order to estimate the position of the target object following a perspective shift, participants need to first encode the position of the target object during encoding, then to compare the encoding and test stimulus to understand how they have moved through space (i.e. to understand the perspective shift which requires self-localization at both encoding and test), and finally to recompute the target object position given their new location in the environment. 
When camera rotations are introduced, the distance to the object and other features in the environment remains the same but the location of the object and other features of the environment on the screen are uniformly offset by the rotation angle. Thus, the relative position of the target object in relation to other features in the environment on the image remains the same despite appearing at a different part of the image. As a result, participants do not really need to self-localize during camera rotations as they can rely on their memory for the object position relative to other nearby features in the environment. Alternatively, they can use the offset in the position of other features in the environment to estimate the position of the target object. However, when camera translations are introduced, the distance between one's own position and other objects changes. Notably, this change is not uniform and depends on the position of the objects. This leads to changes in the vectors and angles between the self and the environmental features, including the to-be-remembered object locations, and therefore to positions these features occupy on the screen. Participants need to consider this new information to understand how they moved through space, and to update the target object position accordingly.

Since camera translations are more difficult to resolve than camera rotations, they introduce more uncertainty about the position of the target object. Consistent with the anchor and adjustment heuristic (Tversky \& Kahneman, 1974), we suggest that due to higher uncertainty following camera translations than rotations, participants exhibit greater reliance on an egocentric anchor (Epley et al., 2004; Gilovich et al., 2000; Keysar et al., 2000). According to the anchor and adjustment heuristic, the anchor is typically adjusted until a plausible response is reached, however, such adjustments are often insufficient (Tversky \& Kahneman, 1974; Quattrone, 1982) such that the response remains biased in the direction of the initial estimate. In our task, the egocentric anchor is the self-to-object vector during encoding. Insufficient adjustment of this egocentric vector on basis of the perspective shift, would result in a systematic shift in object position estimates in the same direction as camera translations 
and rotations. Moreover, in line with our interpretation that camera translation results in greater uncertainty and consequently greater reliance on the anchor, the systematic shift is greater when camera translation rather than camera rotations are introduced.

The idea that uncertainty mediates the reliance on the egocentric anchor is in line with the reduction of the systematic bias introduced by camera translations when additional spatial information (stable environmental cues) was provided in the environment. Specifically, the addition of stable environmental cues is likely to improve the precision with which the object location can be encoded (Cánovas et al, 2011; Chamizo et al., 2011; Kamil \& Chen, 2001;

Ekstrom \& Yonelinas, 2020).Consistent with this account, we found that participants are more precise when environmental cues are available. Additionally, the presence of these cues enriches the spatial structure of the environment and can therefore improve the understanding of the perspective shift. Improvements in the precision with which participants can encode the object location and the understanding of their own position following camera movements is bound to reduce the uncertainty that participants have about object positions at test. In turn, this is likely to reduce the weight that is given to the egocentric anchor during target object position estimation following a perspective shift.

Additional spatial information may not only help to reduce the uncertainty that participants have allowing them to rely less on an egocentric anchor but may also help them to improve the adjustment process. Specifically, additional cues may limit the range of plausible object positions. That is, if the object was between the two columns, then participants can use this information during adjustments to reduce errors as well as the systematic bias in the direction of camera movements.

The finding in our study of a greater detrimental effect of camera translations than rotations on overall performance and on the systematic bias in object position estimate, is inconsistent with the finding from the spatial updating literature that typically shows that 
imagined rotations have a more debilitating effect on performance than imagined translations (Klatzky et al., 1998; Rieser, 1989; Wraga, 2003; Presson \& Montello, 1994). In spatial updating studies greater error is observed during imagined rotations than translations because the latter are less computationally demanding (Rieser, 1989; Presson \& Montello, 1994). For example, Rieser (1989) argued that during imagined translations participants can simply retrieve the stored information from memory. However, for imagined rotations participants either need to recompute the object-to-object relations considering their new orientation or combine the signed self-to-target angle and the signed self-to-observation point angle. Both of those would require additional mental computations to transform the initial encoded representation of object locations.

Also, Presson and Montello (1994) suggested that differences between the imagined rotations and translations in a spatial updating task may be driven by a conflict between actual and imagined heading directions. Specifically, they proposed that humans have a strong tendency to use their immediate heading direction as a primary frame of reference. And in the imagined rotation condition participants need to override this primary frame of reference to adopt an alternative imagined heading direction. Such conflict between reference frames is not present in the translation condition as the actual and imagined heading always remain the same. The lack of conflict between reference frames is also likely to make the updating of selfto-object relations easier (Presson \& Montello, 1994).

In our task, however, the impact of camera rotations and translations is different. Specifically, the object-to-object relations as they are projected on the screen change in the camera translation condition but not in the camera rotation condition. In addition, as noted earlier, the self-to-object relations are uniformly offset in the rotation condition, therefore the new self-to-target object relations can be calculated much easier in conditions when camera rotations are introduced. Conversely, in the translation condition participants need to engage 
in more demanding computations to estimate the new self-to-target object position.

Furthermore, in our task, there is no conflict between heading directions. Participants are shown their new heading direction instead of imagining it. Therefore, their new heading is apparent at both encoding and test. Thus, in our view, the differential impact of rotations and translations between our task and the spatial updating paradigms is responsible for differences in the results.

The experimental design allowed us to investigate how the influence of camera rotations and translations combine during camera movements that include both rotation and translation components to influence participants' performance. We found that a simple linear model with additive inputs of pure rotation and pure translation errors closely matches the empirical data for combined camera movements and provides a significantly better fit than models that are based on errors associated with translations or rotations only. This result suggests that rotation and translation influences do not follow the winner-takes-it-all principle that has been used to explain higher-level cognitive phenomena such as visual attention (Itti and Koch, 2001; Walther \& Koch, 2006) and decision making (Wang, 2002; Furman \& Wang, 2008). Instead, we believe that performance on trials with combined camera translation and rotations results from independent influences of rotations and translations that are linearly combined to produce the observed errors. The linear additive model also explains the smaller errors observed after incongruent camera movements (camera rotates and translates in opposite direction) compared to congruent camera movements (camera rotates and translates in the same direction). Specifically, in incongruent movements, the errors have opposite signs since they are biased in the direction of movement for both camera rotations and translations. Therefore, when the errors are combined, they cancel each other out. In congruent movements the errors for rotations and translations are biased in the same direction and are therefore additive. 
The final aim of Experiment 2 was to investigate how ageing affects the precision with which participants remember the position of objects following a perspective shift and whether older adults are differentially affected by camera rotations and translations as well as by the presence of additional cues in the environment compared to younger adults. Against our predictions, we found that, overall, older adults performed just as well as the younger participants. We did, however, find that older adults benefited less than younger adults from the addition of stable environmental cues. This is in line with our previous work (Segen et al, 2021c) in which we showed that older adults were more biased by the direction of the perspective shift when estimating object displacement directions than younger adults in whom the addition of extra spatial information substantially reduced the systematic bias related to the perspective shift.

One explanation for why older adults benefited less than younger adults from the presence of additional environmental cues is that the presentation of extra cues was not blocked. Instead, trials with and without additional environmental cues were randomly presented. This may have prevented older adults from utilising these additional cues due to problems in switching strategies to use additional information when it was available (for review on strategy switching in navigation and aging see Colombo et al., 2017). Instead, older adults may have relied only on the information that was available across all trials. Additionally, our previous eye-tracking research using similar tasks suggests that older adults have a preference towards encoding object locations in a room using more distal room based cues such as posters, rather than encoding the spatial layout of more proximal object cues distributed in the room (Segen at al., 2021a, 2021b). This preference may further contribute to older adults not utilizing the additional spatial information in the room when it was available.

In line with our predictions, we found that older adults were more affected by camera translation than younger adults and displayed a greater bias in the direction of camera 
translations when estimating target object positions. Also, compared to younger participants, older adults exhibited larger absolute angular errors when camera translations were introduced. The larger bias in the direction of camera translation in older adults may be driven by greater uncertainty stemming from less precise encoding of object locations (Dai et al., 2016; Pertzov et al., 2015; Nilakantan et al., 2018; Segen et al., 2021a; McAvan et al., 2021) and difficulties in spatial perspective-taking in older adults (Segen et al., 2021a; Montefinese et al., 2015; Watanabe, 2011; Inagaki et al., 2002), which cause them to rely more on an egocentric anchor. Yet, contrary to our predictions, older adults did not display a bias in the direction of camera rotations, unlike younger adults who were affected by camera rotations. It is not clear from the current data why older participants were less affected by camera rotations than younger adults. This could be the focus of future studies.

The differential response to camera rotations and translation in older compared to younger adults may also explain the larger absolute angular errors in incongruent camera movements. Specifically, if errors for a specific rotation (i.e. right) are linearly combined with errors for a specific translation (i.e. left) then in younger adults, who are more biased by rotations, the rotation and translation errors are have different signs and when combined the angular errors are reduced in incongruent trials. However, since older adults show only very small systematic rotation errors, when combined with translation errors, the overall errors do not reduce as much as with younger participants in the incongruent trials. Note that the agespecific differences in angular errors related to camera rotations and translations are very small compared to the main effects of camera translation and rotations that we report in the current study. More research is needed to understand the role of ageing in mediating the effects camera rotations and translation have on memory for object locations.

\subsection{General Summary}


To summarise, in the present study we evaluated people's ability to estimate object positions following a perspective shift. In Experiment 1, we replicated (Segen et al., 2021d) a systematic shift in position estimates in the same direction as the perspective shift. In Experiment 2 we investigated the contribution of camera rotations and translations to this bias and showed that translations are largely responsible for causing a systematic bias in object location estimation. Camera translations introduced a greater change in the relations between own position and the object as well as other features in the environment compared to rotations. We believe that those greater changes lead to increased uncertainty regarding the position of an object in the environment which results in greater reliance on egocentric anchors leading to the systematic bias in errors in the same direction as translations. We also show that the influence of camera translations is influenced by both environmental properties and individual differences (age-related difference), such that the bias was larger in less informative environments and in older adults whose abilities to remember object locations have been shown to decline (Montefinese et al., 2015; Muffato et al., 2019; Hilton et al., 2020; Segen et al., 2021a). Lastly, this is the first study to show that the influence of camera rotations and translations on participants' performance is guided by a linear additive process.

\subsection{References}

Bates, D., Kliegl, R., Vasishth, S., \& Baayen, H. (2015). Parsimonious mixed models. arXiv preprint arXiv:1506.04967.

Cánovas, R., León, I., Serrano, P., Roldán, M. D., \& Cimadevilla, J. M. (2011). Spatial navigation impairment in patients with refractory temporal lobe epilepsy: evidence from a new virtual reality-based task. Epilepsy \& Behavior, 22(2), 364-

369.https://doi.org/10.1016/j.yebeh.2011.07.021

Chamizo, V. D., Artigas, A. A., Sansa, J., \& Banterla, F. (2011). Gender differences in landmark learning for virtual navigation: The role of distance to a goal. Behavioural processes, 88(1), 20-26. https://doi.org/10.1016/j.beproc.2011.06.007 
Colombo, D., Serino, S., Tuena, C., Pedroli, E., Dakanalis, A., Cipresso, P., \& Riva, G. (2017). Egocentric and allocentric spatial reference frames in aging: A systematic review. Neuroscience \& Biobehavioral Reviews, 80, 605-621.

Diwadkar, V. A., \& McNamara, T. P. (1997). Viewpoint dependence in scene recognition. Psychological science, 8(4), 302-307.

Easton, R. D., \& Sholl, M. J. (1995). Object-array structure, frames of reference, and retrieval of spatial knowledge. Journal of Experimental Psychology: Learning, Memory, and Cognition, 21(2), 483.

Ekstrom, A. D., \& Yonelinas, A. P. (2020). Precision, binding, and the hippocampus: Precisely what are we talking about?. Neuropsychologia, 138, 107341.

Epley, N., Keysar, B., Van Boven, L., \& Gilovich, T. (2004). Perspective taking as egocentric anchoring and adjustment. Journal of personality and social psychology, 87(3), 327.

Epstein, R., Harris, A., Stanley, D., \& Kanwisher, N. (1999). The parahippocampal place area: Recognition, navigation, or encoding? Neuron, 23(1), 115-125. https://doi.org/10.1016/S0896-6273(00)80758-8

Evensmoen, H. R., Lehn, H., Xu, J., Witter, M. P., Nadel, L., \& Håberg, A. K. (2013). The anterior hippocampus supports a coarse, global environmental representation and the posterior hippocampus supports fine-grained, local environmental representations. Journal of cognitive neuroscience, 25(11), 1908-1925.

Franz, G. (2005). An empirical approach to the experience of architectural space (Doctoral dissertation, Bauhaus-Universität Weimar, Germany).

Furman, M., \& Wang, X. J. (2008). Similarity effect and optimal control of multiplechoice decision making. Neuron, 60(6), 1153-1168.

Gilovich, T., Medvec, V. H., \& Savitsky, K. (2000). The spotlight effect in social judgment: an egocentric bias in estimates of the salience of one's own actions and appearance. Journal of personality and social psychology, 78(2), 211.

Hamburger, K., \& Röser, F. (2014). The role of landmark modality and familiarity in human wayfinding. Swiss Journal of Psychology. 
Hartley, T., Bird, C. M., Chan, D., Cipolotti, L., Husain, M., Vargha-Khadem, F., \& Burgess, N. (2007). The hippocampus is required for short-term topographical memory in humans. Hippocampus, 17(1), 34-48.

Hegarty, M., \& Waller, D. (2004). A dissociation between mental rotation and perspective-taking spatial abilities. Intelligence, 32(2), 175-191.

Hilton, C., Muffato, V., Slattery, T. J., Miellet, S., \& Wiener, J. (2020). Differences in Encoding Strategy as a Potential Explanation for Age-Related Decline in Place Recognition Ability. Frontiers in Psychology, 11.

Inagaki, H., Meguro, K., Shimada, M., Ishizaki, J., Okuzumi, H., \& Yamadori, A. (2002). Discrepancy between mental rotation and perspective-taking abilities in normal aging assessed by Piaget's three-mountain task. Journal of Clinical and Experimental Neuropsychology, 24(1), 18-25.

Itti, L., \& Koch, C. (2001). Computational modelling of visual attention. Nature reviews neuroscience, 2(3), 194-203.

Kamil, A. C., \& Cheng, K. (2001). Way-finding and landmarks: the multiple-bearings hypothesis. Journal of Experimental Biology, 204(1), 103-113.

Keysar, B., Barr, D. J., Balin, J. A., \& Brauner, J. S. (2000). Taking perspective in conversation: The role of mutual knowledge in comprehension. Psychological Science, 11(1), 32-38.

King, J. A., Burgess, N., Hartley, T., Vargha-Khadem, F., \& O'Keefe, J. (2002). Human hippocampus and viewpoint dependence in spatial memory. Hippocampus, 12(6), 811-820.

Klatzky, R. L. (1998). Allocentric and egocentric spatial representations: Definitions, distinctions, and interconnections. In Spatial cognition (pp. 1-17). Springer, Berlin, Heidelberg.

Langnes, E., Vidal-Piñeiro, D., Sneve, M. H., Amlien, I. K., Walhovd, K. B., \& Fjell, A. M. (2019). Development and decline of the hippocampal long-axis specialization and differentiation during encoding and retrieval of episodic memories. Cerebral Cortex, 29(8), 3398-3414. 
Levine, M., Jankovic, I. N., \& Palij, M. (1982). Principles of spatial problem solving. Journal of Experimental Psychology: General, 111(2), 157.

McAvan, A., Du, Y., Oyao, A., Doner, S., Grilli, M., \& Ekstrom, A. (2021). Older Adults Show Reduced Spatial Precision but Preserved Strategy-Use During Spatial Navigation Involving Body-Based Cues. Frontiers in Aging Neuroscience, 13, 129.

Mathôt, S., Schreij, D., \& Theeuwes, J. (2012). OpenSesame: An open-source, graphical experiment builder for the social sciences. Behavior research methods, 44(2), 314-324.

Montefinese, M., Sulpizio, V., Galati, G., \& Committeri, G. (2015). Age-related effects on spatial memory across viewpoint changes relative to different reference frames. Psychological research, 79(4), 687-697.

Nadel, L., Hoscheidt, S., \& Ryan, L. R. (2013). Spatial cognition and the hippocampus: the anterior-posterior axis. Journal of cognitive neuroscience, 25(1), 22-28. https://doi.org/10.1162/jocn a 00313

Nilakantan, A. S., Bridge, D. J., VanHaerents, S., \& Voss, J. L. (2018). Distinguishing the precision of spatial recollection from its success: Evidence from healthy aging and unilateral mesial temporal lobe resection. Neuropsychologia, 119, 101-106.

Pertzov, Y., Heider, M., Liang, Y., \& Husain, M. (2015). Effects of healthy ageing on precision and binding of object location in visual short term memory. Psychology and aging, 30(1), 26.

Postma, A., Kessels, R. P., \& van Asselen, M. (2004). The neuropsychology of objectlocation memory. Human spatial memory: Remembering where, 143-160.

Presson, C. C., \& Hazelrigg, M. D. (1984). Building spatial representations through primary and secondary learning. Journal of experimental psychology: Learning, memory, and cognition, 10(4), 716.

Presson, C. C., \& Montello, D. R. (1994). Updating after rotational and translational body movements: Coordinate structure of perspective space. Perception, 23(12), 1447-1455.

Quattrone, G. A. (1982). Overattribution and unit formation: When behavior engulfs the person. Journal of personality and social psychology, 42(4), 593. 
Rieser, J. J. (1989). Access to knowledge of spatial structure at novel points of observation. Journal of Experimental Psychology: Learning, Memory, and Cognition, 15(6), 1157.

Roskos-Ewoldsen, B., McNamara, T. P., Shelton, A. L., \& Carr, W. (1998). Mental representations of large and small spatial layouts are orientation dependent. Journal of Experimental Psychology: Learning, Memory, and Cognition, 24(1), 215.

Sancaktar, I., \& Demirkan, H. (2008). Spatial updating of objects after rotational and translational body movements in virtual environments. Computers in human behavior, 24(6), 2682-2696.

Schmidt, D., Krause, B. J., Weiss, P. H., Fink, G. R., Shah, N. J., Amorim, M. A., ... \& Berthoz, A. (2007). Visuospatial working memory and changes of the point of view in 3D space. Neuroimage, 36(3), 955-968.

Segen, V., Avraamides, M. N., Slattery, T. J., \& Wiener, J. M. (2021a). Age-related differences in visual encoding and response strategies contribute to spatial memory deficits. Memory \& cognition, 49(2), 249-264.

Segen, V., Avraamides, M. N., Slattery, T. J., \& Wiener, J. M. (2021b). Age-related changes in visual encoding strategy preferences during a spatial memory task. Psychological Research, 1-17.

Segen, V., Colombo, G., Avraamides, M., Slattery, T., \& Wiener, J. M. (2021c). Perspective taking and systematic biases in object location memory. Attention, Perception, \& Psychophysics, 1-19.

Segen, V., Colombo, G., Avraamides, M. N., Slattery, T. J., \& Wiener, J. M. (2021d). The role of memory and perspective shifts in systematic biases during object location estimation. bioRxiv

Sulpizio, V., Committeri, G., Lambrey, S., Berthoz, A., \& Galati, G. (2013). Selective role of lingual/parahippocampal gyrus and retrosplenial complex in spatial memory across viewpoint changes relative to the environmental reference frame. Behavioural brain research, $242,62-75$. 
Tversky, A., \& Kahneman, D. (1974). Judgment under uncertainty: Heuristics and biases. science, 185(4157), 1124-1131.

Walther, D., \& Koch, C. (2006). Modeling attention to salient proto-objects. Neural networks, 19(9), 1395-1407.

Wang, R. F., \& Spelke, E. S. (2002). Human spatial representation: Insights from animals. Trends in cognitive sciences, 6(9), 376-382.

Wang, X. J. (2002). Probabilistic decision making by slow reverberation in cortical circuits. Neuron, 36(5), 955-968.

Watanabe, M. (2011). Distinctive features of spatial perspective-taking in the elderly. The International Journal of Aging and Human Development, 72(3), 225-241.

Wraga, M. (2003). Thinking outside the body: An advantage for spatial updating during imagined versus physical self-rotation. Journal of Experimental Psychology: Learning, Memory, and Cognition, 29(5), 993. 